\title{
New achievements in positron and positronium chemistry
}

\author{
Hugh D. Burrows* \\ Departamento de Química, Universidade de Coimbra, 3004-535 Coimbra, Portugal
}

This conference PPC-8 highlighted some of the most important developments in positron and positronium chemistry.

New chemistry was considered in the presentation of J.Y. Jean on the use of intense slow positron beam, while A.P. Mills, Jr. discussed the possibility of chemistry and physics with many positrons. These studies are likely to lead to new atoms and molecules - as was discussed in part by J.R. Mohallem on interaction with noble gas atoms.

A general discussion was made on positronium formation. The connection with radiation chemistry was considered through the presentations of T. Hirade, and S. Stepanov's Blob models, whilst M. Eldrup discussed the past, present and future of Ps trapping.

Of particular relevance to positron and positronium chemistry is the application in structural studies. Spectroscopic, microscopic, scattering and diffraction methods study what is there. In contrast, positron annihilation methods look at what is not there-free volume, making them ideal for studying amorphous, microporous and mesoporous materials.

Particular emphasis was placed on polymer free volume in systems such as polymer nanocomposites (F. Maurer; S. Wang), polyimide films and membranes (K. Ratzke; C. Bas) and generally on amorphous polymers (G. Consolati). In addition, this topic was discussed extensively in a Round Table involving G. Brauer, J.Y. Jean, T. Suzuki, A.L.R. Bug and A. Alam. These considerations were extended to polymerization and reaction studies in the presentations of A. Shimazu (photopolymerization), A. Somoza (setting of epoxy

\footnotetext{
${ }^{*}$ Tel.: + 351239854482 ; fax: + 351239827703

E-mail address: burrows@ ci.uc.pt.
}

resins) and F. Saito (ion irradiated polystyrene). The application of positron and positronium chemistry in studying the glass transition was considered by M.A. Alam in his presentation on glass forming liquids confined in Vycor pores.

Other important amorphous systems which were discussed included grains and powders both with experimental measurements (Y. Kobayashi) and simulation of the behaviour of Ps in void spaces (A.L.R. Bug), while R. Zaleski gave a detailed discussion of the PAL spectral analysis of mesoporous silica. One area of great practical importance involves the application of these techniques to low- $\kappa$ dielectrics and composites. This was discussed in the presentations of R. Suzuki (interconnect materials for interlayer dielectrics in integrated circuits), R. Vallery; S.Eijt; R. Brusa (low- $\kappa$ dielectric films and networks) and M. Fujinami (metallic nanoparticles in dielectric materials).

Other applications in non-metallic solids included studies of free volumes in solids under high pressure (T. Goworek), the effects of morphology-polyamide 6 (M. Debowska), the quenching of Ps by $\mathrm{Fe}$ in silica based glasses (K. Inoue) and the damage evolution in irradiated sapphire (I. Israelashvily). Similarly studies of coatings, films, layered structures and surfaces were considered by K.G. Lynn (porosity of mesoporous dielectric thin films), H. Chunqing ( $\mathrm{Si}$ thin films), P. Mascher, G. Brauer, J. Kuriplach (Si/silicon oxide films and interfaces), S. Mariazzi (W-Si-N thin films), K. Ito (PECVD siloxane films) and C. Hugenschmidt (Al coated PMMA surfaces).

Finally, liquids and condensed multiphase systems were discussed in a series of important communications, considering aspects such as liquids and porous substances (B. Lévay, B. Ganguly), micellar surfactant and polymer systems (G. Duplâtre), polycrystalline 
inorganic systems (V.P. Shantarovich) and supercritical fluids (T. Sekine).

The Workshop acted as an excellent forum to discuss these topics. However, there still remain a number of unanswered questions. One major direction that is being considered involves new chemistry (i.e. what new molecules can we form containing positron or the positronium atom). Another area in which advances are to be expected involves free-volume studies. In particular, can we study pore shape and anisotropy? Also, when we apply these studies to surfaces, whether on thin films, multilayers, biological systems, etc., how well can we carry out depth profiling? As seen from this and previous Workshops, positron and positronium techniques have already been applied to many areas involving soft matter, including micelles and microemulsions. There are, however, a number of other systems, such as nanoparticles, liquid crystals and self-assembly systems, which are, as yet, relatively unexplored. The area of studies with biological systems using these techniques is in its infancy. This must be expanded, and will provide the link between positron annihilation and PET. In particular, understanding of many of the chemical processes involved is likely to be of great importance to the development and interpretation of PET results. Finally, many of the models of positron and positronium behaviour are still at the empirical or, at best, semi-empirical level. Attempts must be made to develop more quantitative models if we are truly to understand the physics involved in these interesting systems.

As can be seen by the Papers in these Proceedings, a number of important advances have been made in our understanding of positron and positronium chemistry, However, we are likely to see active research in these areas, which we believe will be reflected in further developments presented at the ninth Workshop in China in 2008 . 